\title{
May 2015 Critical Care Case of the Month: An Infected Leg
}

\author{
Sandra L. Till DO and Robert A. Raschke MD \\ Banner University Good Samaritan Medical Center \\ Phoenix, AZ
}

\section{History of Present IIIness}

A 46-year-old transferred due to concern for necrotizing fasciitis. One the day prior to transfer purple discoloration was not noted in the lower portion of the left leg. On the day of transfer the leg became more purple, painful, and swollen. She presented to a pain clinic that advised her to go to an emergency room. The emergency room performed arterial Doppler ultrasound, which was normal and transferred her due to concern of necrotizing fasciitis.

\section{Past Medical History, Social History and Family History}

She has a past medical history of fibromyalgia. She had an extensive surgical history including an appendectomy, bladder implant, cholecystectomy, dilatation and curettage, esophageal repair, left femoral artery repair due to a motor vehicle accident, partial hysterectomy, left knee surgery, and several left leg operations with grafting. Family history was non-contributory. The patient was single with two children, and smoked 1-2 packs of cigarettes per day for 30 years. She denied any illicit drugs or alcohol abuse.

\section{Medications \\ - Zolpidem \\ - Warfarin \\ - Furosemide \\ - Potassium Chloride \\ - Morphine sulfate \\ - Gabapentin \\ - Oxycodone \\ - Alprazolam \\ - Ondansetron \\ - Amitriptyline}

\section{Physical Examination}

Vitals signs: Blood pressure 128/85 mm Hg, pulse 86 beat/min, respiratory rate 12 , temperature $36.7^{\circ} \mathrm{C}$, SPO2 $96 \%$ on $2 \mathrm{~L} / \mathrm{min}$ of oxygen.

General: Non-toxic, alert and oriented x3, tearful due to pain.

The remainder of the physical examination was unremarkable except for the left lower extremity (Figure 1). 


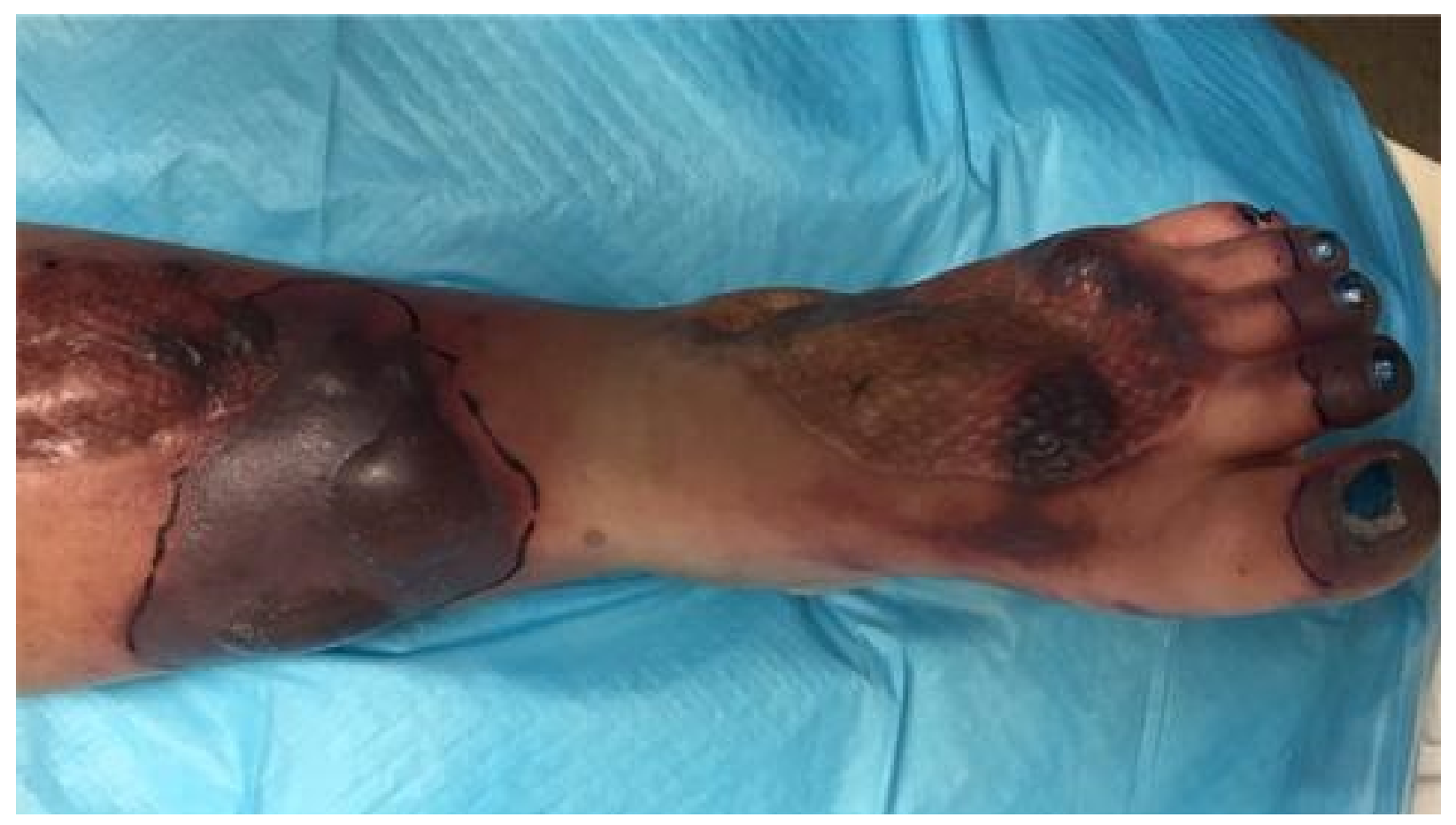

Figure 1. Photograph of the patient's left leg.

Which of the following are appropriate at this time?

1. Blood cultures

2. Complete blood count, c-reactive protein, sodium, creatinine and glucose

3. Surgery consult

4. Wound culture

5. All of the above 


\section{Correct! \\ 5. All of the above}

The patient appears to have an infected leg with possible necrotizing fasciitis and possibly sepsis. Therefore, cultures of the wound and blood, a surgery consult and baseline laboratory studies are all appropriate.

The laboratory evaluation was surprisingly unremarkable except for a white blood cell count of 29,600 cells/microliter with a left shift. The laboratory risk indicator for necrotizing fasciitis was low at 2. No organisms were seen on the gram stain although atypical cells were noted. The wound and blood cultures showed no growth. The surgery consultation recommended broad-spectrum antibiotics and following the patient.

Which of the following should be done next?

1. Ask the pathologist to look at the Gram stain

2. Hyperbaric oxygen

3. Insist on surgical debridement

4. 1 and 3

5. All of the above 


\section{Correct! \\ 1. Ask the pathologist to look at the Gram stain}

The surgeons were reluctant to operate because of the low laboratory risk indicator for necrotizing fasciitis although up to $10 \%$ of the patients with necrotizing fasciitis have a risk indicator of $<6$ (1). The laboratory risk indicator for necrotizing fasciitis is a scoring system using CRP, white blood count, hemoglobin, sodium, creatinine, and glucose to classify patients into risk categories. A score of 2 placed her at low risk. Hyperbaric oxygen, which can be used for necrotizing fasciitis, seems overly aggressive with the diagnosis unclear. However, when the pathologist reviewed the Gram stain, promyelocytes were seen.

Which of the following is appropriate?

1. Bone marrow aspiration/biopsy

2. Examination of the peripheral blood for promyelocytes

3. Fluorescent in situ hybridization (FISH) for RARA

4. 1 and 3

5. All of the above 


\section{Correct! \\ 5. All of the above}

Flow cytometry and careful examination of the peripheral smear revealed the presence of promyelocytes (Figure 2).

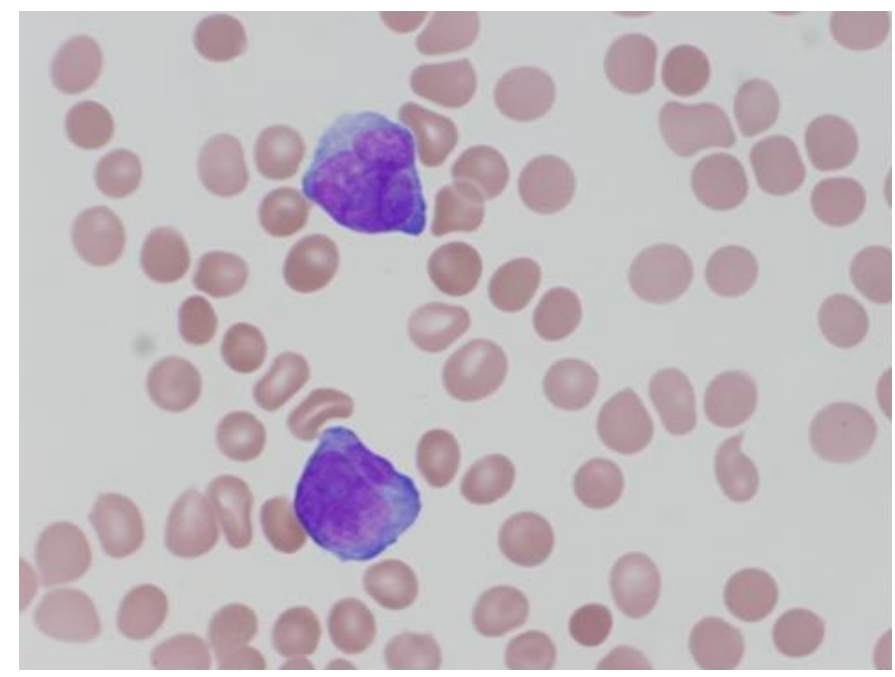

Figure 2. Peripheral smear showing two blasts.

The bone marrow aspiration biopsy resulted in a dry tap. However, the core biopsy showed hypercellular bone marrow compatible with acute promyelocytic leukemia (Figure 3).

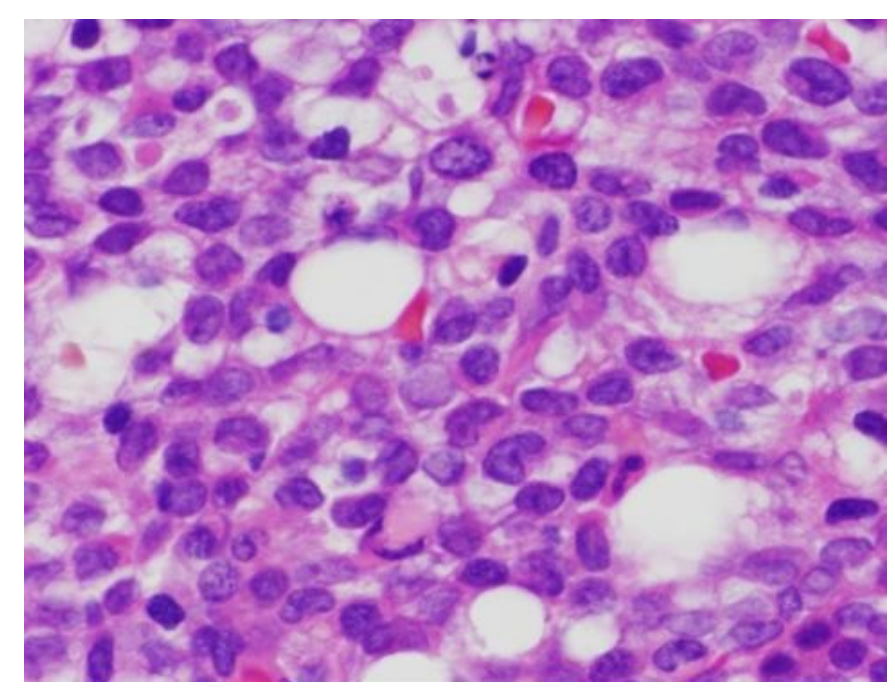

Figure 3. High power view of the core bone marrow biopsy showing hypercellularity with multiple promyelocytes.

FISH for the chromosomal translocation involving the retinoic acid receptor alpha (RARa or RARA) gene on chromosome 17 on the peripheral blood was positive. 
Which of the following are true regarding acute promyelocytic leukemia (APL)?

1. APL can be a manifestation of arsenic poisoning

2. APL is a common leukemia

3. APL is a medical emergency and treatment should be initiated with all-trans retinoic acid

4. 1 and 3

5. All of the above 


\section{Correct! \\ 3. APL is a medical emergency and treatment should be initiated with all-trans retinoic acid}

APL is unique among leukemia's due to its sensitivity to all-trans retinoic acid (ATRA; tretinoin), the acid form of vitamin A (2). Treatment with ATRA allows induces the terminal differentiation of the leukemic promyelocytes, after which these differentiated malignant cells undergo spontaneous apoptosis on their own. APL is a medical emergency with a high rate of early mortality often due to hemorrhage from coagulopathy. Treatment with ATRA should be initiated as soon as the diagnosis is suspected.

APL accounts for $5-20 \%$ of cases of acute myelogenous leukemia with only $600-800$ new cases per year in the US. APL is not a rapidly proliferative acute leukemia. The course of the preclinical phase is unclear. Presentation is usually late in the course with life-threatening catastrophic bleeding. Bleeding is caused by degranulation of tissue factor, which leads to disseminated intravascular coagulation (DIC), and release of leukocyte proteases that leads to hypofibrinogenemia. This constitutes a true hematologic emergency that many intensivists will eventually have to recognize and treat. At time of diagnosis the bone marrow is typically replaced by $100 \%$ malignant promyelocytes leading to severe anemia, thrombocytopenia and neutropenia. Only a small number of APL cells may be present in the blood.

ATRA alone is capable of inducing remission but it is short-lived in the absence of concurrent "traditional" chemotherapy (2). The standard of treatment for concurrent chemotherapy is arsenic trioxide. Chemotherapy with ATRA and arsenic trioxide results in a clinical remission in approximately $90 \%$ of patients. ATRA syndrome or cytokine storm syndrome is a potentially fatal complication of induction chemotherapy for APL (3). It occurs in $25 \%$ of patients with APL during induction therapy and is caused by the release inflammatory vasoactive cytokines leading to capillary leak. Symptoms include dyspnea, edema, unexplained fever, hypotension, pulmonary hemorrhage, respiratory failure, renal failure, hepatic failure, and cerebral edema. If suspected, dexamethasone should be initiated while chemotherapy is continued.

The patient was initiated on ATRA chemotherapy and developed improvements in her leg examination along with blood counts within days of starting the therapy (Figure 4). 


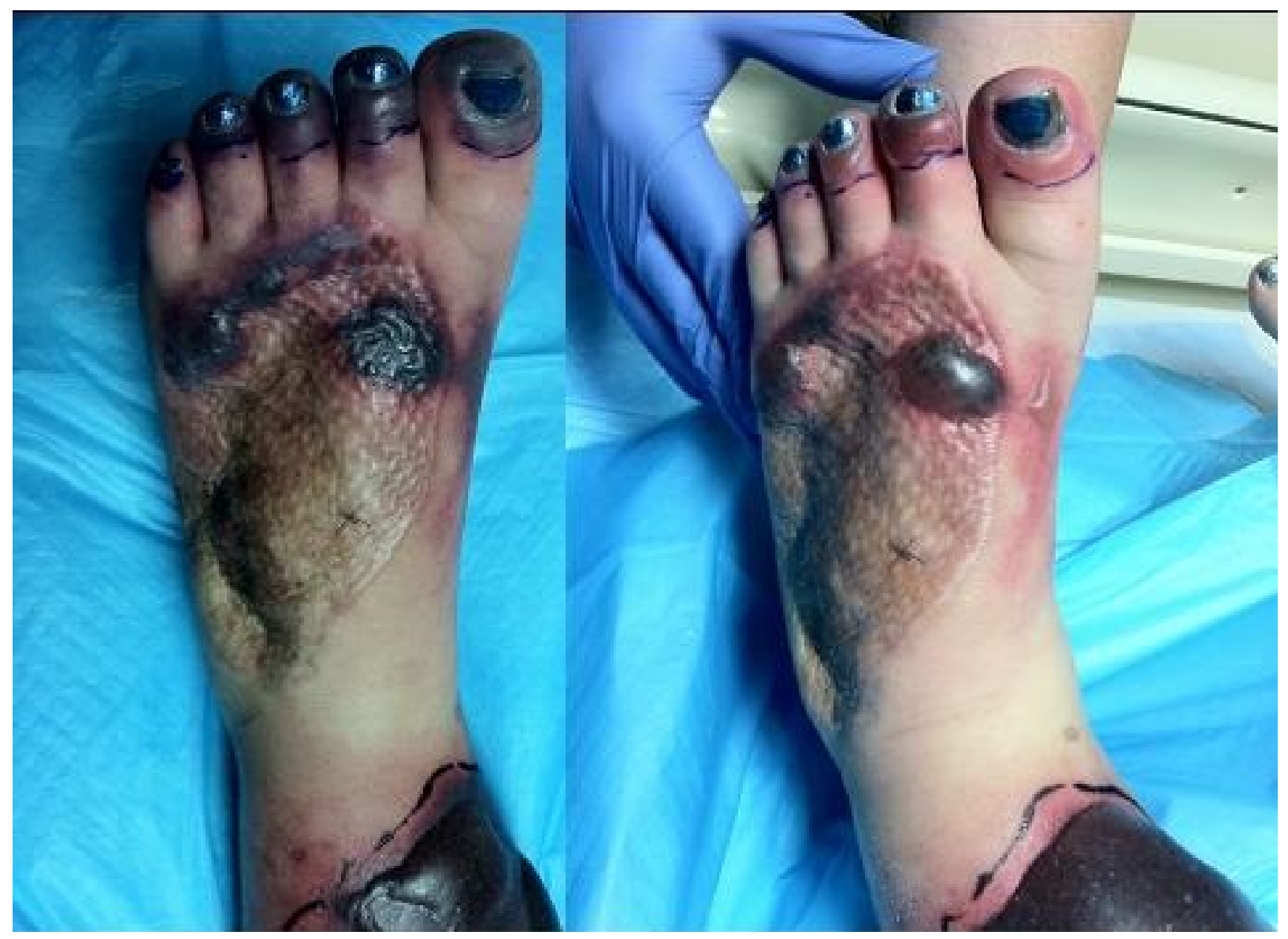

Figure 4. Before (left) and after (right) pictures. The picture on the right was taken 3 days after ATRA was started.

\section{References}

1. Misiakos EP, Bagias $G$, Patapis $P$, Sotiropoulos $D$, Kanavidis $P$, Machairas $A$. Current concepts in the management of necrotizing fasciitis. Front Surg. 2014;1:36. [CrossRef] [PubMed]

2. Breccia M, Lo Coco F. Thrombo-hemorrhagic deaths in acute promyelocytic leukemia. Thromb Res. 2014;133 Suppl 2:S112-6. [CrossRef] [PubMed]

3. Sanz MA, Montesinos P. How we prevent and treat differentiation syndrome in patients with acute promyelocytic leukemia. Blood. 2014;123(18):2777-82. [CrossRef] [PubMed] 